\section{Haplotype roundup}

Several recently published papers address limitations of existing genome sequencing approaches in distinguishing haplotypes. By starting with a very small number of cells and using a special sample preparation workflow to deliver a single copy of a chromosome to a well in a microtiter plate, Peters et al. report a wholegenome sequencing strategy that can differentiate the paternal chromosome from the maternal.

Two reports by Fan et al. and Kitzman et al. also describe noninvasive haplotype analysis of a fetus by sequencing fetal DNA circulating in the mother's bloodstream. Whereas Kitzman et al. required knowledge of both the mother's and the father's genome to sequence the fetal genome, the strategy of Fan et al. dispenses with the need for a paternal sample.

Peters, B. et al. Accurate whole-genome sequencing and haplotyping from 10 to 20 human cells. Nature 487, 190-195 (2012)

Fan, H.C. et al. Non-invasive prenatal measurement of the fetal genome. Nature advance online publication, doi:10.1038/nature11251, 4 July 2012.

Kitzman, J. et al. Noninvasive whole-genome sequencing of a human fetus. Sci. Transl. Med. 4, 137ra76 (2012).

\section{Who's eating who in the human gut?}

Stern et al. use a tailored algorithm to exploit the presence of the bacterial CRISPR (clustered regularly interspaced short palindromic repeat) immune system to detect bacteriophages that prey on human gut bacteria. The CRISPR system integrates fragments of incoming viral or plasmid DNA into the bacterial genome to form CRISPR loci (barcodes of mobile elements that have infected a bacterium), then packages transcribed CRISPR mRNA sequences into a protein complex that patrols the cell and degrades incoming matching foreign DNA. Stern et al. extract CRISPR barcodes from the MetaHIT data set ( $>500$ million bp of microbiome genes from 124 people) and use these barcodes to pull out almost 1,000 phage genomes from MetaHIT contigs. By scanning several human gut microbiome data sets, they show that almost $80 \%$ of these phages are shared by individuals around the world. They also used the CRISPR sequences to match gut phages to the bacterial species they infect, based on the presence of CRISPR barcodes in individually sequenced human gut bacteria. Finally, they identify individuals with high and low phage abundance. Although we cannot yet link phage-mediated modulation of gut microbiota composition to health or disease, this analysis is a step in the right direction. (Genome Res. advance online publication, doi:10.1101/gr.138297.112, 25 June 2012)

\section{Shear drug delivery}

Systemic administration of thrombolytic drugs can restore disrupted blood flow caused by ischemic stroke or pulmonary embolism. However, when diffusing freely throughout the body, these agents can also cause

Written by Christine Borowski, Susan Jones, Jason Kreisberg \& Craig Mak dangerous bleeding. Korin et al. devise a new therapeutic strategy to target thrombolytic activity to occluded vessels. Knowing that fluid shear stress can be orders of magnitude higher in constricted compared with normal vessels, the authors fabricate microscale nanoparticle aggregates cued to break apart into smaller nanoparticles upon exposure to pathologically high local shear stress. Subjected to lower drag forces than their microscale precursors, the smaller nanoparticles can adhere more efficiently to substrates such as endothelial cells and clots. When coated with the thrombolytic drug tissue plasminogen activator (tPA), these platelet biomimetics accumulated in regions of vascular occlusion and eroded clots in mouse models of arterial thrombosis and pulmonary embolism. Because they are effective at 100 -fold lower doses than soluble $\mathrm{tPA}$, are rapidly cleared from the circulation and can in theory be coated with a variety of thrombolyic agents, these shear-activated nanotherapeutics hold promise for treating a range of disorders associated with vascular obstruction. (Science advance online publication, doi:10.1126/ science.1217815, 5 July 2012)

\section{FIAsH of insight into HIV}

Super-resolution microscopy techniques can now routinely achieve a resolution of $20-50 \mathrm{~nm}$, but for a protein to be imaged, it typically must be fluorescently labeled with an antibody or fused to a fluorescent protein. Imaging unperturbed, functional proteins is difficult using these labeling methods because antibodies can only penetrate fixed and permeabilized cells introducing potential artifacts, and fluorescent proteins can have a deleterious effect on function. Lelek $e t$ al. adapt fluorescein arsenical helix binder (FlAsH) labeling to super-resolution microscopy, achieving a resolution of $\sim 30 \mathrm{~nm}$. The cell-permeable FlAsH reagent binds in either live or fixed cells to an engineered tetracysteine motif. The authors use FlAsH to image an engineered yet still functional variant of the integrase protein of HIV both in free virions and during infection. They observe that during viral transit through the cytoplasm, integrase is not released into the cell and instead remains located within the viral capsid. Only after the incoming virion docks at the nuclear pore are smaller clusters of fluorescent signal seen in the nucleus, potentially containing individual integrase molecules bound to the viral genome. This study demonstrates the utility of a small, nondisruptive label for super-resolution imaging. Because of the small size of the tetracysteine label and the relative brightness of the dye, this approach may allow for single nanometer resolution imaging in the future. (Proc. Natl. Acad. Sci. $109,8564-8569,2012)$

\section{Complex mammalian cell circuit design}

Last year, two groups used collections of modified yeast or bacterial cells to create biological systems that could respond to multiple inputs in a manner analogous to how a digital circuit processes information. Ausländer et al. now show that such behavior can be introduced into a single mammalian cell. The authors engineer human cells to enable them to perform one of the fundamental tasks of information processing systems-adding and subtracting single bits of information. In elementary school, children learning to add multidigit numbers are taught the concept of 'carrying' forward (e.g., to compute 29 plus 29, add the 9s, carry 1 forward and add the 2 s plus the carried 1). Conceptually, the circuit of Ausländer et al. can perform a similar carrying operation, allowing it to add two single-digit binary numbers. In practical terms, the circuit takes as input two small molecules, the antibiotic erythromycin and the plant metabolite phloretin, and produces as outputs yellow and red fluorescent protein. The circuit marks a new standard of complexity achieved in synthetic biology. (Nature 487, 123-127, 2012) 